\title{
Influence of different amount of Au on the wetting behavior of PDMS membrane
}

\author{
Jiang-Tao Feng • Ya-Pu Zhao
}

Published online: 21 July 2007

(C) Springer Science + Business Media, LLC 2007

\begin{abstract}
Polydimethylsiloxane (PDMS) has been widely used as a base material for bio-MEMS/NEMS devices. It is difficult for PDMS to transfer and spread aqueous solution as a kind of highly hydrophobic material. Therefore, surface modification is necessary for PDMS to make it hydrophilic. In this paper, a method of hydrophilization of PDMS surface is proposed. Gold is sputtered to the PDMS substrate by sputter coater in different average thicknesses. Relationship between the average thickness of gold on the PDMS substrate and the contact angle of the surface was studied. It was found that even gold of average thickness less than $1 \mathrm{~nm}$ can result in about $25^{\circ}$ change of contact angle. AFM is also used to get topographic information of PDMS surface coated with gold. Three cases are classified with different amount of Au: (1) Heterogeneous zone; (2) Transition zone; (3) Film zone. For heterogeneous zone, a simple model about heterogeneous phase wetting is put forward to interpret this phenomenon.
\end{abstract}

Keywords PDMS · Wetting · Hydrophilic modification . Heterogeneous phase

\section{Introduction}

Polydimethylsiloxane (PDMS) elastomer is the most widely used silicon-based organic polymer in the fabrication of various microfluidic devices, rapid prototyping and nanolithography. PDMS has both advantages and disadvantages.

\section{J.-T. Feng $\cdot$ Y.-P. Zhao $(\triangle)$}

State Key Laboratory of Nonlinear Mechanics,

Institute of Mechanics, Chinese Academy of Sciences,

15 Beisihuanxi Road,

Beijing 100080, China

e-mail: yzhao@imech.ac.cn
The well-known advantages of PDMS include: it is optically transparent, chemically inert, non-toxic and nonflammable. Especially due to its biocompatibility, flexibility and simple fabrication using soft lithography, it has been applied to many bio-MEMS and other fields as substrate and microfluidic devices (McDonald and Whitesides 2002). On the other hand, PDMS has its own disadvantages in spite of many advantages. The surface of PDMS is inherently hydrophobic, and it is difficult to transfer and spread aqueous solutions (Bodas and Khan-Malek 2006), and this disadvantage, of course, hinders the wide application of PDMS for microfluidic devices.

A number of techniques have been utilized to hydrophilize the PDMS surface, including physical, chemical or a combination of both (Bodas and Khan-Malek 2006). Among these methods, oxygen plasma is the most widely employed to make the hydrophilization of PDMS surface. Nevertheless, this technique suffers from a short lifetime before hydrophobic recovery (Seo and Lee 2006; Hillborg et al. 2001; Hillborg et al. 2000; Hillborg and Gedde 1998). To overcome the difficulty of quick hydrophobic recovery, twostep modification has been employed by some groups (Gilmor et al. 2002; Bodas and Khan-Malek 2006) using various gases. Similar to PDMS, SU-8 is another widely used bio-MEMS material and is also highly hydrophobic, hydrophilic modification of SU-8 by oxygen plasma and its stability have been studied very recently (Walther et al. 2007).

Other surface modification methods such as accumulation or depletion of surfactant in PDMS (Seo and Lee 2006), silanization, adsorption of polymer or protein, and lipid coating (Barker et al. 2000; Liu et al. 2000; Linder et al. 2001; Makamba et al. 2003) have also been demonstrated.

As an alternative method of hydrophilization of PDMS surface, this paper studied the influence of different amount of $\mathrm{Au}$ sputtered on the PDMS surface on its wetting 
behavior. Besides, electric conductivity of $\mathrm{Au}$ is much better than PDMS and DNA can hybridize on the $\mathrm{Au}$ surface to fabricate bio-sensors (Wu et al. 2001). PDMS deposited with gold may be applied to bio-MEMS or electric devices. Relationship between the average thickness of gold on the PDMS substrate and the contact angle of the surface was studied in the present paper.

\section{Experimental}

\subsection{Sample preparation}

PDMS membrane (polydimethylsiloxane, Sylgard 184, Dow Corning, USA; ratio of the base to curing agent= 10:1) which we used was vacuumed for an hour to remove the trapped air-bubbles, and then was cured about $20 \mathrm{~h}$ at $75^{\circ} \mathrm{C}$ in a class- 1,000 clean room. The thickness of PDMS layer is about $1.82 \mathrm{~mm}$.

Sputter Coater (BAL-TEC, SCD 050) was used to coat PDMS substrate with gold at 0.1 Torr using $20 \mathrm{~mA}$ current while the substrate temperature is $20^{\circ} \mathrm{C}$. The thickness of gold can be read from the Sputter Coater. In the sputter coater, the thickness of gold is measured by a quartz measurement. Gold was deposited at $0.02 \mathrm{~nm} / \mathrm{s}$, the average thicknesses of gold are $0.4,0.8,1.2,1.6,2.0,2.5,3.5,5.0$, and $10.0 \mathrm{~nm}$ in our experiments, respectively. To make comparison and check the repetition of the experiments, three groups of samples denoted by 1, 2, and 3 were prepared. The temperature and relative humidity of the experiments are $17^{\circ} \mathrm{C}$ and $32 \%$ for group $1,25^{\circ} \mathrm{C}$ and $30 \%$ for group $2,22.5^{\circ} \mathrm{C}$ and $31 \%$ for group 3 . The relative error of average thickness increases with the decrease of the average gold thickness. It is well known that very large residual stress will be generated in the gold films when the films are thick enough (Mertens et al. 2007). Since PDMS membrane is very soft, a Si plate is used to support it as shown in Fig. 1. Without Si plates supporting, moving the samples will make the gold films break into pieces. This may also relate to the poor adhesion between gold and PDMS (Lee et al. 2005; Lim et al. 2006).

\section{gold}

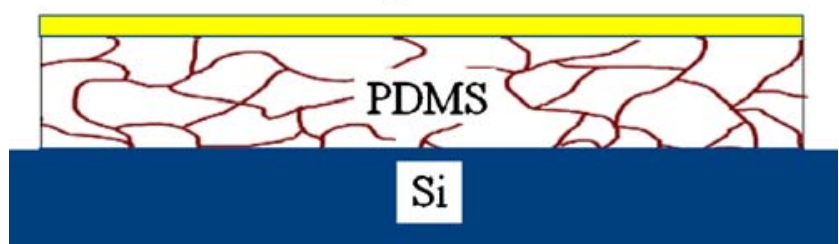

Fig. 1 The sample structure. Average thickness of gold is on the order of $1 \mathrm{~nm}$. The thicknesses of PDMS membrane and Si plate are about 1.82 and $1.0 \mathrm{~mm}$, respectively
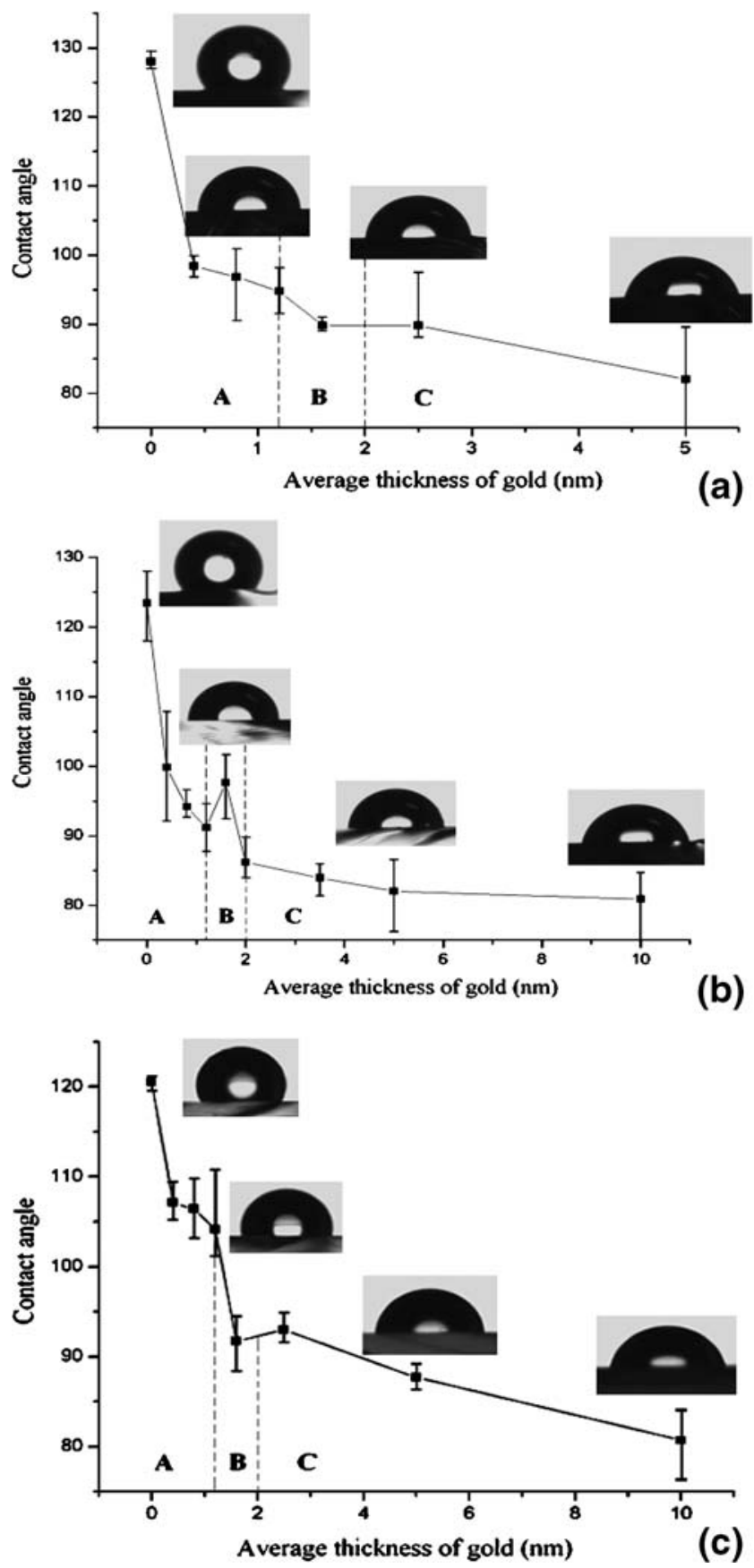

Fig. 2 Average thickness of gold on the PDMS substrate versus contact angle. (a) For group 1, (b) for group 2, and (c) for group 3. Three cases are classified with different amount of Au: $(A)$ heterogeneous zone; $(B)$ transition zone; $(C)$ film zone

\subsection{Results and discussion}

Contact angles were measured with the OCA20 system (precision $\pm 0.1^{\circ}$ ) from Dataphysics, Germany. A sessile drop method was used to determine the contact angle of water on different surfaces. An average of six readings was considered as contact angle of the surface under study. Our experimental results are shown in Fig. 2. From Fig. 2, it is 
Table 1 Change of contact angle with time

\begin{tabular}{|c|c|c|c|c|c|c|c|c|c|}
\hline \multirow[t]{2}{*}{ Time } & \multicolumn{9}{|c|}{ Average thickness } \\
\hline & $0 \mathrm{~nm}\left({ }^{\circ}\right)$ & $0.4 \mathrm{~nm}\left({ }^{\circ}\right)$ & $0.8 \mathrm{~nm}\left({ }^{\circ}\right)$ & $1.2 \mathrm{~nm}\left({ }^{\circ}\right)$ & $1.6 \mathrm{~nm}\left({ }^{\circ}\right)$ & $2.0 \mathrm{~nm}\left({ }^{\circ}\right)$ & $2.5 \mathrm{~nm}\left({ }^{\circ}\right)$ & $5.0 \mathrm{~nm}\left({ }^{\circ}\right)$ & $10.0 \mathrm{~nm}\left({ }^{\circ}\right)$ \\
\hline 1 st day & $120.5 \pm 0.9$ & $107.1 \pm 2.1$ & $106.4 \pm 3.4$ & $104.1 \pm 2.9$ & $91.7 \pm 2.8$ & $93.0 \pm 1.9$ & $90.5 \pm 0.2$ & $87.7 \pm 1.5$ & $80.7 \pm 3.4$ \\
\hline 2nd day & $116.8 \pm 2.0$ & $105.1 \pm 1.6$ & $103.9 \pm 2.0$ & $99.6 \pm 2.5$ & $95.3 \pm 4.1$ & $95.2 \pm 4.6$ & $94.4 \pm 2.3$ & $88.8 \pm 1.1$ & $80.1 \pm 2.2$ \\
\hline 3rd day & $115.2 \pm 3.7$ & $104.3 \pm 0.5$ & $104.1 \pm 0.7$ & $100.6 \pm 3.0$ & $94.3 \pm 2.0$ & $91.5 \pm 2.9$ & $97.6 \pm 2.3$ & $89.9 \pm 0.2$ & $89.2 \pm 0.9$ \\
\hline 4th day & $119.5 \pm 2.5$ & $101.0 \pm 2.4$ & $102.3 \pm 1.7$ & $97.7 \pm 3.4$ & $96.7 \pm 2.3$ & $91.1 \pm 2.5$ & $93.9 \pm 0.6$ & $89.2 \pm 2.5$ & $90.8 \pm 1.9$ \\
\hline
\end{tabular}

found that the results of the three sample groups have the resemble curves for contact angle versus average thicknesses of gold. It is shown from Fig. 2 that contact angle decreases gradually with the increase of the average thickness of gold, which means that the hydrophilization of PDMS surface increases. At first, the decrease of the contact angle is abrupt when the average thickness of gold is less than $1.0 \mathrm{~nm}$. It turns to be mild when the average thickness of gold is larger than $2.0 \mathrm{~nm}$. The contact angle of clean PDMS membrane is $128 \pm 1.4^{\circ}$ (group 1), $123.3 \pm 3.1^{\circ}$ (group 2), and $120.5 \pm 0.9^{\circ}$ (group 3), respectively. When the average thickness of the gold is close to $0.4 \mathrm{~nm}$ which means that only some $\mathrm{Au}$ islands are formed on the PDMS surface, the contact angle reduces to $98.5 \pm 1.4^{\circ}$ (group 1), $99.8 \pm 4.2^{\circ}$ (group 2), and $107.1 \pm 2.1^{\circ}$ (group 3). It is seen that even gold of average thickness less than $1.0 \mathrm{~nm}$ can bring a decrease of about $25^{\circ}$ in contact angle. When average thickness of the gold film increases to $5.0 \mathrm{~nm}$, the contact angle decreases to $81.4 \pm 4.6^{\circ}$ (group 1), $82.0 \pm 4.3^{\circ}$ (group 2), and $87.7 \pm 1.5^{\circ}$ (group 3), respectively. The abrupt increase at the data point of $1.6 \mathrm{~nm}$ in Fig. 2(b) does not appear in data of other groups. The abrupt increase may be caused by some occasional factors.

The stability of hydrophilic modification of PDMS surface is very important. Samples (group 3) were used to test the stabilization. The change of contact angle of group 3 for 4 days was observed and relationship between the change of contact angle and time is summarized in Table 1. From Table 1, it is seen the contact angle of the sample does not recover when the average thickness of gold is less than $5.0 \mathrm{~nm}$. The stability of hydrophilic behavior of our samples is better than other methods like treating by oxygen plasma and chemical immersion (Mata et al. 2005). When the average thickness of gold is $10 \mathrm{~nm}$, the contact angle recovers from 80.7 to $90.8^{\circ}$ in the fourth day.

The surface of PDMS membrane with sputtered gold was observed by stereomicroscope (Olympus S $2 \times 12$ ) and was shown in Fig. 3. From Fig. 3 we can see the surface color changes a lot in appearance with the increase of the amount of gold. The influence of the big tensile residual stress can be seen from Fig. 3 (h), and the $\mathrm{Au}$ film was ruptured due to the tensile residual stress.
Optical measurements were performed with a spectrophotometer (Unico, UV-2802h). The transmittance T of the samples was tested with various wavelengths, and the transmittance is defined as

$T \equiv \frac{I}{I_{0}} \times 100 \%$

where $I$ and $I_{0}$ are the transmitted and the incident intensities, respectively. The experimental results are shown in Fig. 4. The gold on the PDMS surface affects the transparency of PDMS membrane dramatically.

To know the reason of those changes in contact angles, atomic force microscopy (AFM) (VEECO, Co. USA) was used to study the surface. Surface of PDMS is very flat (see Fig. 5). The surface of PDMS coated with gold is scanned in tapping mode and Fig. 6 are presented. When the average thickness of the gold film is 0.4 or $0.8 \mathrm{~nm}$, the sputtered gold on the PDMS surface is in fact individual particles. The Au particles coalesce with each other when the average thickness increases to $1.2 \mathrm{~nm}$, and the gold film begins to form. When the average gold thickness increases to $2.0 \mathrm{~nm}$, we can see the gold particles cover the whole surface of PDMS. Three cases are classified with different amount of $\mathrm{Au}$ (Fig. 6):

- Heterogeneous zone: when the amount of sputtered Au is not sufficient to form complete Au film on the PDMS surface, $\mathrm{Au}$ islands are formed on the PDMS surface. The surface is treated as a heterogeneous one, and the wetting property is described by the modified Cassie equation (Eq. 6) with the surface roughness taken into account;

- Transition zone: when the average Au film is between 1.2 and $2.0 \mathrm{~nm}, \mathrm{Au}$ film starts to form somewhere and $\mathrm{Au}$ islands grow to touch each other;

- Film zone: Au film will be formed on PDMS surface when the average thickness is larger than $2.0 \mathrm{~nm}$. The gold particles cover the whole surface of PDMS.

This classification is different from other situations of sputtered gold. Sputtered gold on other materials such as 
Fig. 3 (a), (b), (c), (d), (e), (f), (g), and (h) denote surface of PDMS coated with gold of average thickness $0.4,0.8,1.2$, $1.6,2.0,2.5,5.0$, and $10.0 \mathrm{~nm}$, respectively

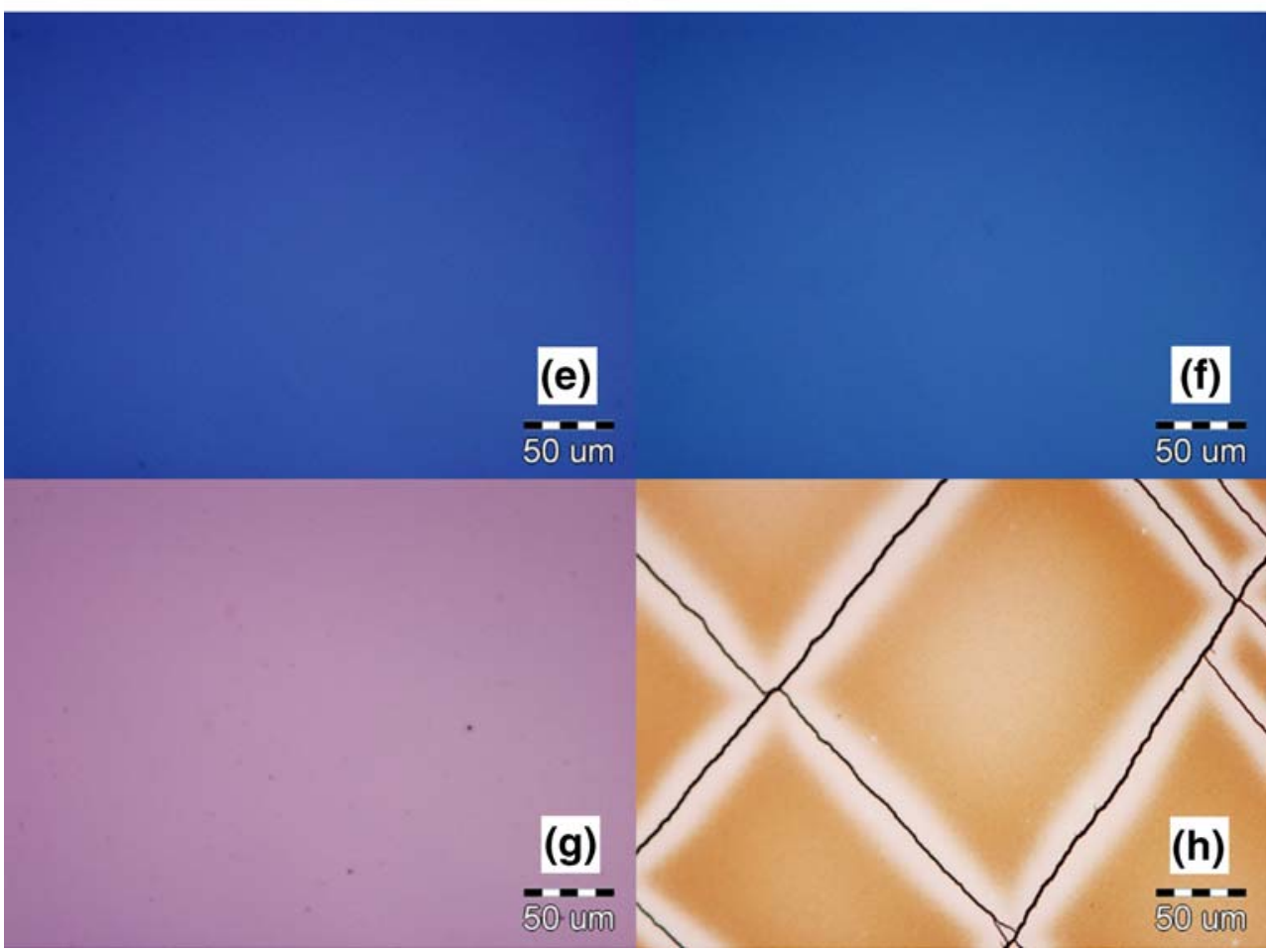

polyethyleneterephthalate (PET) has been reported (Svorcik et al. 2002, 2004; Deki et al. 1999), the sputtered gold layer on PET surface was thought to start to be a continuous film when average thickness of gold is larger than $10 \mathrm{~nm}(50 \mathrm{~s}$, $0.02 \mathrm{~nm} / \mathrm{s}$ ).

\section{Modeling}

Since we get the AFM pictures of the PDMS surface coated with gold. We want to find why gold of average thickness less than $1.0 \mathrm{~nm}$ can lower contact angle near $25^{\circ}$. For the 


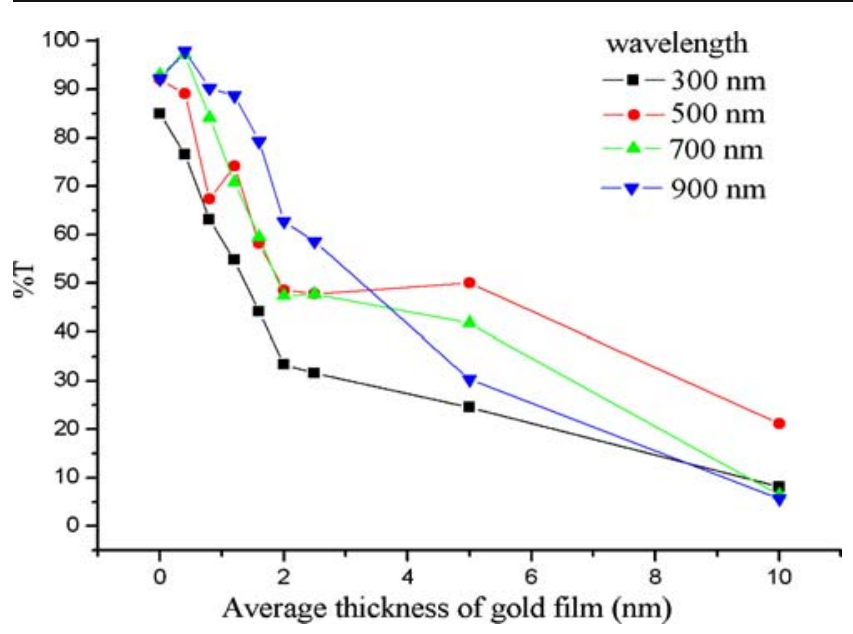

Fig. 4 Plots of transmittance $T$ versus average thickness of gold in various wavelengths

surface is still heterogeneous when average of thickness of gold is less than $1.0 \mathrm{~nm}$, we use modified Cassie equation (Eq. 6) to evaluate it. For transition zone and film zone, there is still no model to estimate them.

When a droplet is placed on a solid surface, it remains as a drop having a definite contact angle between the liquid and solid phases or spreads across the surface to form a wetting film. Young stated the contact angle $\theta_{0}$ for a smooth surface at the tri-phase contact line (Young 1805), composed by the surface tensions in the solid-vapor $\left(\gamma_{\mathrm{sv}}\right)$, liquid-vapor $\left(\gamma_{\mathrm{lv}}\right)$ and solid-liquid $\left(\gamma_{\mathrm{sl}}\right)$ interfaces, the Young's equation is

$\cos \theta_{0}=\frac{\gamma_{\mathrm{sv}}-\gamma_{\mathrm{sl}}}{\gamma_{\mathrm{lv}}}$.

The origin of surface tension $\gamma$ can be explained at the molecular level (de Gennes et al. 2004).
The topography patterns on surfaces play a significant effect on wetting in nature (Neinhuis and Barthlott 1997; Gao and Jiang 2004), such as the configuration of lotus leaves and other kinds of ultra-hydrophobic surfaces (Ren et al. 2003). The roughness effect is classically described by Wenzel's model (Wenzel 1936) which introduces a roughness factor $R$ for correction, Wenzel's model is related to the Young equation for smooth surface by the equation as follows

$\cos \theta=R \cos \theta_{0}$,

where $\theta$ is the contact angle for rough surface, the roughness factor gives the ratio of actual to projected solid-liquid contact area:

$R=\frac{A_{\mathrm{sl}(\text { true })}}{A_{\mathrm{sl}(\text { apparent })}}$.

If the surface is heterogeneous, an alternative model for the heterogeneous surface was developed by Cassie (1948). The composite contact angle $\theta_{c}$ for the heterogeneous surface is expressed by

$\cos \theta_{\mathrm{c}}=f_{1} \cos \theta_{1}+f_{2} \cos \theta_{2}$,

where $f_{1}$ and $f_{2}$ are the fractions of the surface occupied by surface types having contact angles $\theta_{1}$ and $\theta_{2}$. Since in our experiment, there are two phases which are gold and PDMS and the roughness of the surface must be taken into account. So we extend Eq. 5 to

$\cos \theta_{\mathrm{c}}=f_{1} \cos \theta_{1}+R f_{2} \cos \theta_{2}$,

the subscripts 1 and 2 denote those quantities associated with PDMS and gold particles, respectively. Here we take $\theta_{1}=123^{\circ}$ and $\theta_{1} \approx 50^{\circ}$ (Notsu et al. 2005).
Fig. 5 Clean PDMS surface. The roughness asperities are less than $3.0 \mathrm{~nm}$

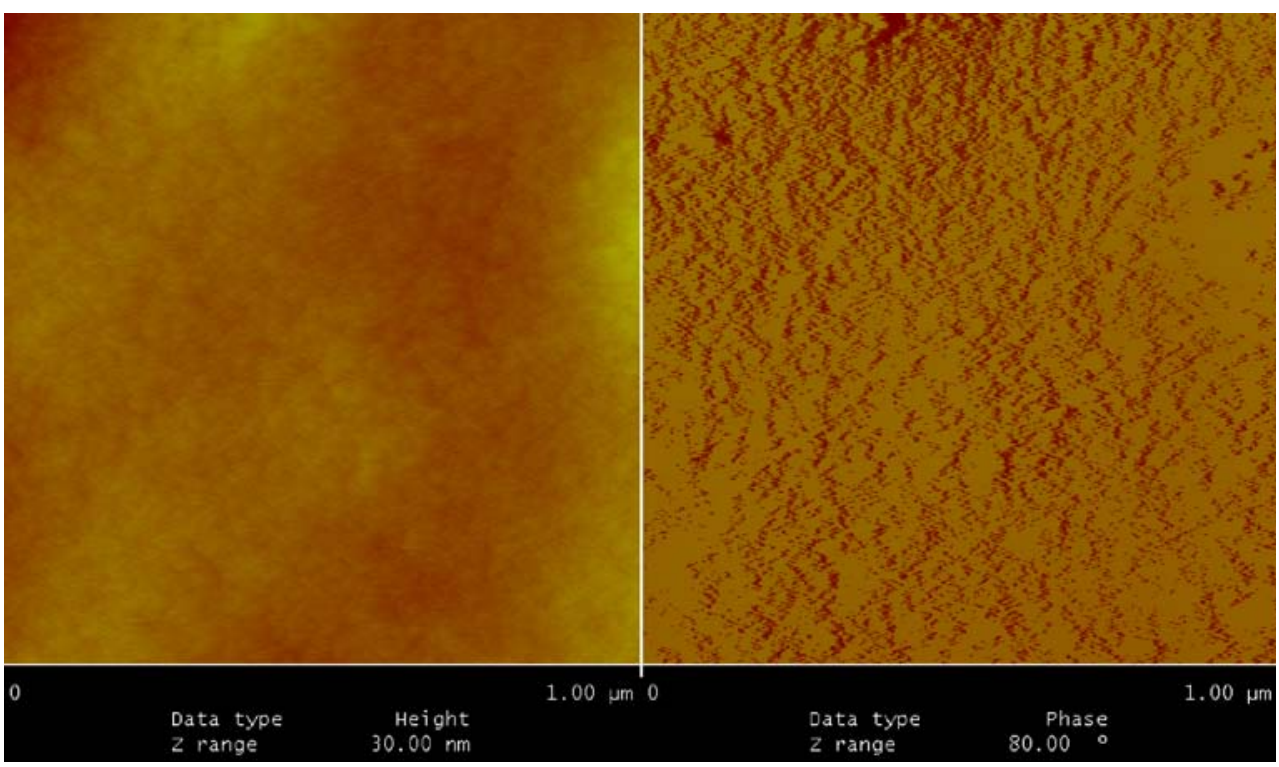



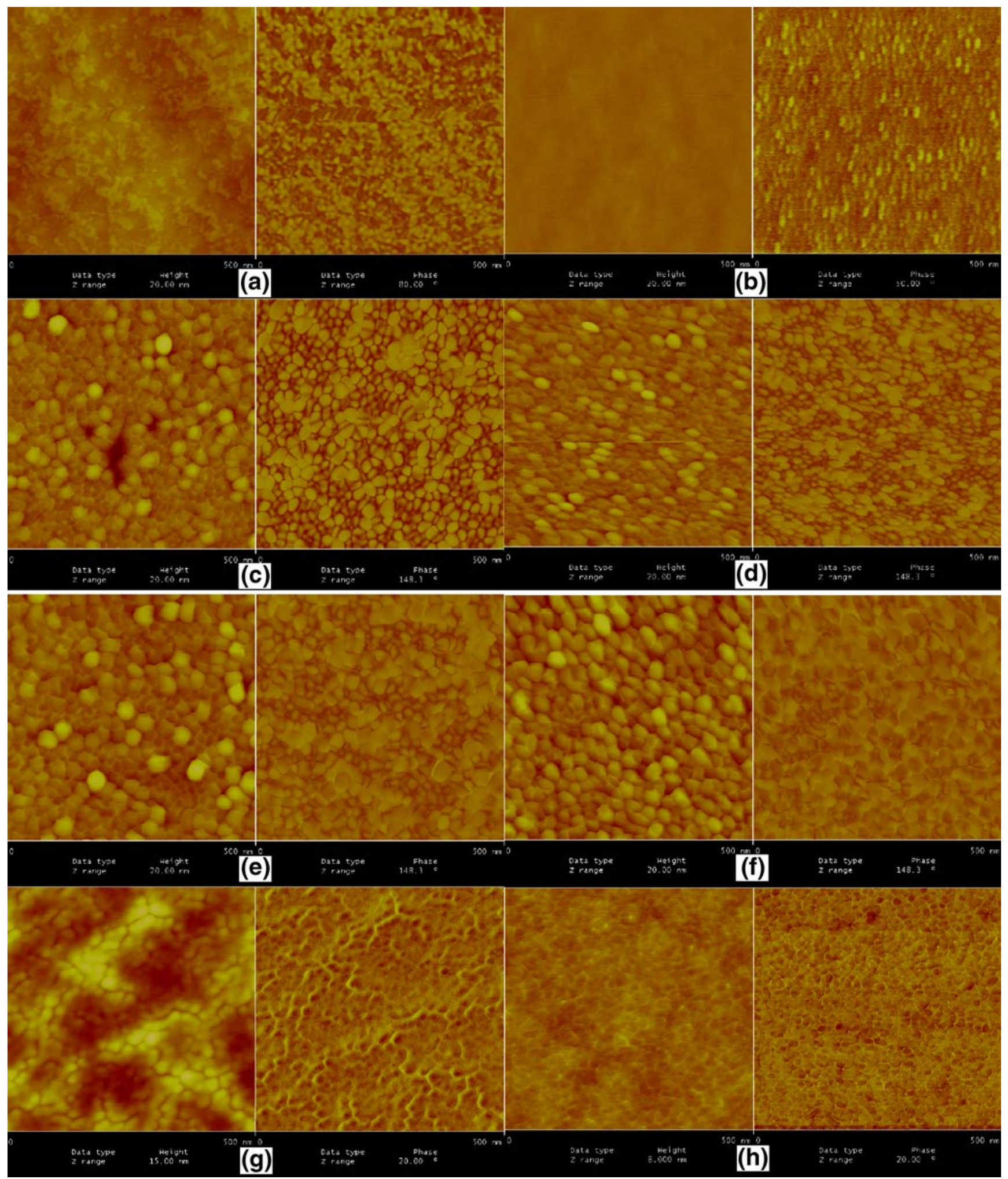

Fig. 6 PDMS surface coated with gold. (a), (b), (c), (d), (e), (f), (g), and (h) denote surface of PDMS coated with gold of average thickness 0.4, $0.8,1.2,1.6,2.0,2.5,5.0$, and $10.0 \mathrm{~nm}$, respectively. Data types are height (left) and phase (right)

For simplicity and without loss of generality, it is reasonable to assume that the gold particles illustrated in Fig. 6(a,b) on the PDMS surface are ellipsoids. It is measured that the three axes of the ellipsoids are 17.0,
$12.4,2.0 \mathrm{~nm}$ (when average thickness of $\mathrm{Au}$ is $0.4 \mathrm{~nm}$ ) and 22.3, 13.1, $1.68 \mathrm{~nm}$ (when average thickness of $\mathrm{Au}$ is $0.8 \mathrm{~nm}$ ). An average of five readings was considered as size of gold particles under study. The surface area of the 


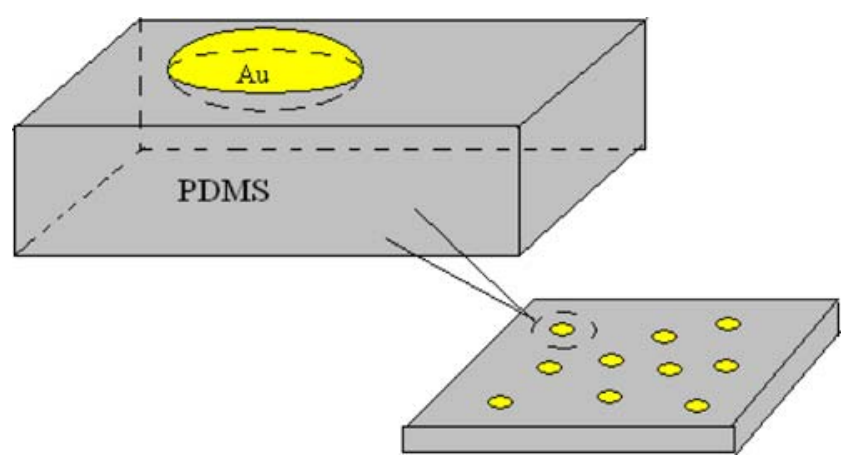

Fig. 7 Sketch map about distributing of gold particles when average thickness of gold film is 0.4 or $0.8 \mathrm{~nm}$

ellipsoid is given by an approximate equation as

$$
S=2 \pi \sqrt{a^{2} b^{2}+b^{2} c^{2}+c^{2} a^{2}+\frac{1}{3}\left(a^{2} b c+b^{2} a c+c^{2} a b\right)},
$$

where $a, b$ and $c$ are three semi-axes of the ellipsoid. Because only part of the gold particle surface contacts with the PDMS substrate while the rest is bare to the air. For the proportion of bare part of gold particle is hard to be measured exactly and the third axis is much shorter than other axes, as a first order approximation and without loss of generality, it is reasonable to assume the bare part is about half of the total, illustrated as Fig. 7. Then the roughness factor is given by

$R=\frac{S}{2 A_{2}}$,

where $A_{2}$ is the projected area of the ellipsoid. We will find $f_{1}, f_{2}$ by

$f_{1}=\frac{A_{1}}{A_{1}+A_{2}}, f_{2}=\frac{A_{2}}{A_{1}+A_{2}}$,

where $A_{1}=\frac{4 \pi a b c}{3 h}-\pi a b, A_{2}=\pi a b$, and it is obvious that $f_{1}+f_{2}=1$.

The comparison between the theoretical model and the experimental measurements is given in Table 2. From Table 2 we can see that the result of this model is in good agreement with the experimental result when average thickness of gold is $0.4 \mathrm{~nm}$. For $0.8 \mathrm{~nm}$, the model shows a discrepancy. It should be noted that the above model is

Table 2 Comparison between model and experiment (sample group 2)

\begin{tabular}{lll}
\hline Average thickness $(\mathrm{nm})$ & Theory $\left(^{\circ}\right)$ & Experiment $\left({ }^{\circ}\right)$ \\
\hline 0.4 & 102.5 & $99.8 \pm 4.2$ \\
0.8 & 72 & $94.2 \pm 2.6$ \\
\hline
\end{tabular}

just an approximate one, many factors are neglected such as surface roughness of PDMS and deviation of the actual gold particle shape from ellipsoid, and so on.

\section{Conclusions}

A novel method of hydrophilization of PDMS surface is proposed. Gold is sputtered to the PDMS surface by sputter coater in different average thicknesses. The influence of $\mathrm{Au}$ average thickness on the wetting behavior of sample surface was studied experimentally with the measurement of contact angle variations. With the increase of average thickness of gold, the contact angle decreases gradually. The contact angle changes from $128.0 \pm 1.3^{\circ}$ (group 1), $123.3 \pm 3.1^{\circ}$ (group 2), and $120.5 \pm 0.9^{\circ}$ (group 3), to $81.4 \pm 4.6^{\circ}$ (group 1), $82.0 \pm 4.3^{\circ}$ (group 2), and $87.7 \pm 1.5^{\circ}$ (group 3) when the average thickness of gold changes from 0.4 to $5.0 \mathrm{~nm}$. Gold of average thickness less than $1.0 \mathrm{~nm}$ on PDMS surface can bring a change about $25^{\circ}$ in the contact angle of PDMS surface. The wetting model for heterogeneous surface was used to interpret such phenomenon. The approximate theoretical values can explain the experimental results in a certain extent. Different micro-features of samples have been scanned by AFM. The effect of this hydrophilic modification method can last longer time than other methods like treating by oxygen plasma and chemical immersion (Mata et al. 2005).

Acknowledgments This work was supported by the National Basic Research Program of China (973 Program, Grant No. 2007CB310504), National Natural Science Foundation of China (NSFC, Grant No. 10225209), key project from Chinese Academy of Sciences (Grant No. KJCX-SW-L2). The AFM experimental assistance from Dr. Hui-Ling $\mathrm{Li}$ is appreciated. The valuable comments by anonymous referees are gratefully acknowledged.

\section{References}

S.L.R. Barker, D. Ross, M.J. Tarlov, M. Gaitan, L.E. Locascio, Anal. Chem. 72, 5925 (2000)

B. Bodas, C. Khan-Malek, Microelectron. Eng. 84, 1277 (2006)

A.B.D. Cassie, Discuss. Faraday Soc. 3, 11 (1948)

P.G. de Gennes, F. Brochard-Wyart, D. Quéré, Capillarity and Wetting Phenomena: Drops, Bubbles, Pearls, Waves. (Springer, New York, 2004)

S. Deki, K. Sayo, T. Fujita, A. Yamada, S. Hayashi, J. Mater. Chem. 9, 943 (1999)

X. Gao, L. Jiang, Nature 432, 36 (2004)

S. Gilmor, B. Larson, J. Braun, C. Mason, L. Barba, F. Denes, M. Lagally, in Second Annual International IEEE EMBS Conference, vol. 51 (Wisconsin, USA, 2-4 May, 2002)

H. Hillborg, U.M. Gedde, Polymer 39, 1991 (1998)

H. Hillborg, J.F. Ankner, U.M. Gedde, G.D. Smith, H.K. Yasuda, K. Wikstrom, Polymer 41, 6851 (2000)

H. Hillborg, M. Sandelin, U.M. Gedde, Polymer 42, 7349 (2001) 
K.J. Lee, K.A. Tosser, R.G. Nuzzo, Adv. Func. Mater. 15, 557 (2005)

K.S. Lim, W.J. Chang, Y.M. Koo, R. Bashir, Lab Chip 6, 578 (2006)

V. Linder, E. Verpoorte, W. Thormann, N.F. de Rooij, H. Sigrist, Anal. Chem. 73, 4181 (2001)

H. Makamba, J.H. Kim, K. Lim, N. Park, J.H. Hahn, Electrophoresis 24, 3607 (2003)

Y. Liu, J.C. Fanguy, J.M. Bledsoe, C.S. Henry, Anal. Chem. 72, 5939 (2000)

A. Mata, A.J. Fleischman, S. Roy, Biomed. Microdevices 7, 281 (2005)

J.C. McDonald, G.M. Whitesides, Acc. Chem. Res. 35, 491 (2002)

J. Mertens, M. Calleja, D. Ramos, A. Tarýn, J. Tamayo, J. Appl. Phys. 101, 034904 (2007)

C. Neinhuis, W. Barthlott, Ann. Bot. 79, 667 (1997)

H. Notsu, W. Kubo, I. Shitanda, T. Tatsuma, J. Mater. Chem. 15, 1523 (2005)
S.L. Ren, S.R. Yang, Y.P. Zhao, T.X. Yu, X.D. Xiao, Surf. Sci. 546, 64 (2003)

V. Svorcik, V. Rybka, M. Maryska, M. Spirkova, J. Zehentner, V. Hnatowicz, Eur. Polym. J. 40, 211 (2004)

V. Svorcik, J. Zehentner, V. Rybka, P. Slepicka, V. Hnatowicz, Appl. Phys. A 75, 541 (2002)

J. Seo, S.L. Lee, Sens. Actuators, B, Chem. 119, 192 (2006)

F. Walther, P. Davydovskaya, S. Zürcher, M. Kaiser, H. Herberg, A.M. Gigler, R.W. Stark, J. Micromech. Microeng. 17, 524 (2007)

R.N. Wenzel, Ind. Eng. Chem. 28, 988 (1936)

G. Wu, H. Ji, K. Hansen, T. Thundat, R. Datar, R. Cote, M.F. Hagan, A.K. Chakraborty, A. Majumdar, Proc. Natl. Acad. Sci. USA 98, 1560 (2001)

T. Young, Philos. Trans. R. Soc. Lond. 95, 65 (1805) 